\title{
Comparative analysis of elementary andedge-preserving spatial filters in noise removal - a comprehensive study
}

\author{
K. Sivakumar ${ }^{1}$, Sakthiraam. $B^{2}$, Santosh Snehal. $V^{3}$, Yogashivasankarri. $S^{4}$ \\ ${ }^{1}$ Asst. Prof., Department of Electronics and Communication Engineering \\ Rajalakshmi Institute of Technology, Chennai. \\ ${ }^{2}$ Undergraduatestudents, Department of Electronics and Communication Engineering \\ Rajalakshmi Institute of Technology, Chennai. \\ sivakumar.k@ritchennai.edu.in ${ }^{1}$, sakthiraam.b.2018.ece@ritchennai.edu.in², \\ santhoshsnehal.v.2018.ece@ritchennai.edu.in ${ }^{3}$, yogashivasankarri.2018.ece@ritchennai.edu.in ${ }^{4}$
}

\begin{abstract}
Preserving the edges and information is one of the main purposes of edge-preserving filters. That is, they're employed to smooth a picture, and minimize halos, phantoms, and edge blur over the edge. They have a nonlinear relationship between one thing and another. Bilateral filters, anisotropic diffusion filters, directed filters, and trilateral filters are all types of example filters. The filter family may be used in a wide range of image processing tasks, such as denoising, video abstraction, demosaicing, optical flow estimation, stereo matching, tone mapping, style transfer, relighting, and others. The paper gives a clear description of edge preserving filters, from the heat diffusion equation in ancient times to the present, explaining their numerous applications and detailing their numerous uses. Additionally, mathematical analysis is included, as well as efficient and optimized implementations. The focus is on preserving the boundaries, spikes, and canyons, and the information is given clearly and in detail. Finally, it offers a realistic representation of efficient implementation, as well as a comprehensive research scope for future hardware implementation.
\end{abstract}

\section{INTRODUCTION}

Noise reduction is a frequently sought consequence in image processing, particularly to offer a good viewing experience for humans. However, it is also helpful for enabling further processing stages, such as segmentation or feature recognition [9]. This paper analyzes the performance of several simple and edge-preserving spatial filters with noise reduction applications. The spatial image filters [10] are broadly categorized as (i) basic image filtering, which includes the Gaussian filter, median filter, order-statistic filter, local standard deviation of image filter, and local range of image filter, among others. (ii) Edge-Preserving Filtering, such as Bilateral filtering, Anisotropic diffusion filtering, Guided filtering, and so on. (iii) Texture Filtering, such as the Gabor filter bank; (iv) Integral Image Domain Filtering, such as the 2-D integral image filter; and (v) Frequency Domain Filtering, such as FIR window-based filters. This study compares edge preserving filters at various noise levels on both medical and natural images.

The rest of the paper is organized as follows. Section II, a briefsabout spatial filter Section 3 Experimental result for various noise level, section 4 result and discussion, finally section concludes the overall work of this paper.

Key words: Guided filter, non-local means, bilateral, Anisotropic Filter.

\section{SPATIAL FILTERS}

\subsection{Image-Guided filter}

Edge-aware Image filtering is achieved using the guided filter technique. Several real-world applications of the guided filter have been observed, including image editing apps for smartphones and stereo reconstruction.It is a high-speed filter [1].Let $I_{g}$ be the guidance image, $I_{i}$ be the input image and $I_{o}$ be the output image, then the model can be

$$
I_{o}=\alpha I_{g}+\beta
$$

for all pixels in an image. $\alpha, \beta$ real coefficient. The out $I_{o}$ is calculated with window, $W$, of size $r$. 
The Coefficient $\alpha, \beta$ are calculated by minimizing the error between the input $I_{i}$ and the out put $I_{o}$.

$$
\begin{aligned}
\alpha & =\frac{\frac{1}{|W|} \sum_{\text {over } W}\left(\mathrm{I}_{\mathrm{g}} I_{i}-\mu \mathrm{I}_{\mathrm{i}}\right)}{\sigma^{2}+\varepsilon} \\
\beta & =I_{i}-\alpha \mu
\end{aligned}
$$

Where $\sigma$ and $\mu$ are the variance and mean of the input image $I_{i} . \varepsilon$ is small factor which is used to control the smoothness of the output image.The filtered image is given by

$$
\mathrm{I}_{\mathrm{o}}=\alpha I_{i}+\beta
$$

\subsection{Non-local means-filter.}

The objective of any noise removal techniques it to retrieve actual image $u(i)$ from the observed image $v(i)$. The observed image $v(i)$ can be given as

$$
v(i)=u(i)+n(i)
$$

where $n(i)$ is the noise added with each pixel in the image $u(i)$.

According to [2] the non-local mean is estimated as the sum of scaled pixels of observedimage $v$.

$$
\text { Non - local mean }\{[v(\mathrm{i})]\}=\sum S(i, j) v(j)
$$

Whereweights $S(i, j)$ depend on the correlation between the $i^{\text {th }}$ and $j^{\text {th }}$ pixels. The scaling parameter has to full fill the following conditions
(i) $0 \leq S(i, j) \leq 1$ and
(ii) $\sum S(i, j)=1$.

To make the observed image as close as the original is essential in many of the image processing and analysis algorithms. Estimating the noise variance will help to minimize the error between $u$ and $v$. The mask in [2] is construed from two simple masks $L_{1}$ and $L_{2}$. Which is given as

\begin{tabular}{|l|l|l|}
\hline 0 & 1 & 0 \\
\hline 1 & -4 & 1 \\
\hline 0 & 1 & 0 \\
\hline
\end{tabular}

\begin{tabular}{|l|l|l|}
\hline 1 & 0 & 1 \\
\hline 0 & -4 & 0 \\
\hline 1 & 0 & 1 \\
\hline
\end{tabular}

The mask to estimate the noise with zero mean and variance $\sigma_{n}$ is calculates from $L_{1}$ and $L_{2}$ is given by, $\mathrm{N}=2\left(\frac{1}{2} L_{2}-L_{1}\right)$

\begin{tabular}{|r|r|r|}
\hline 1 & -2 & 1 \\
\hline-2 & 4 & -2 \\
\hline 1 & -2 & 1 \\
\hline
\end{tabular}

The variance of the noise in Iis 


$$
\sigma_{n}^{2}=\frac{1}{36(M-2)(N-2)} \sum_{\text {image } I}(I(x, y) * N)^{2}
$$

The limitation of [3] is that it uses one multiplication for each of the pixels. Where $\mathrm{M}, \mathrm{N}$ represent the number of row and columns of the input image and $\mathrm{N}$ is the mask.

\subsection{Bilateral Filter.}

The bilateral filtering combined the range filtering and the domain, thusimplementing both geometric and photometric localitywithin single filtering technique. According to [4], the bilateral filtering is defined as

$$
I_{o}(x)=K^{-1}(x) \iint f(x) * c(y, x) * s(f(y), f(x)) d y
$$

The normalization factor $K$ is given by

$$
K=\int_{-\infty}^{\infty} \int_{-\infty}^{\infty} c(y, x) s(\mathbf{f}(y), \mathbf{f}(\mathbf{x})) d y
$$

Where $x$ is the neighborhood center, $y$ is a nearby pixel, $I_{0}$ is the output picture, $I_{i}$ is the input image, and $s$ is the similarity factor. Because the bilateral filter incorporates the good aspects of both the domain component and the range filter in a proper mix, the filter retains sharp edges.

\subsection{Nonlinear Anisotropic Filtering}

The filtering method proposed in [5] used in the post-processing of the medical images mainly to reduce the acquisition time and to reduce the overall spatial volume. Further this method The procedure beats the limitations of the conventional filtering also retains the fine details which are essential in the case of medical image analysis. The two distinct diffusion functions proposedby[5] is

$$
\begin{aligned}
& c_{1}(\bar{x}, t)=\exp \left(-\left(\frac{|\nabla I(\bar{x}, t)|}{\mathcal{K}}\right)^{2}\right) \\
& c_{2}(\vec{x}, t)=\frac{1}{1+\left(\frac{|\nabla I(\bar{x}, t)|}{\kappa}\right)^{1+\alpha}} \mid \alpha>0 .
\end{aligned}
$$

Where, $I(\bar{x}, t)$ - image intensity, $\nabla$ - spatial derivative operator, $K$ - is the parameter that is chosen according to the level of the noise present in the image $u$.

\subsection{Fast local Laplacian filter.}

The goal of [5] is to overcome constraints such as sluggish interactive editing speed, unknown connection to other filters, and limited manipulation and tone mapping of bilateral and local Laplacian filters. The listed steps are used to achieve fast filtering. (i) Compute the Gaussian pyramid of $I$. (ii) sample the intensity range, (iii) Compute the remapped images and it Laplacian pyramids, (iv)Linearly interpolate, (v)Stop the output.

\subsection{Local entropy filter.}

The texture of the input image may be described using entropy, which is a statistical measure of unpredictability. The normalized histogram counts are represented by $\mathrm{p}$, and entropy is defined as sum(p.*log2(p)). Entropy based filtering finds application in segmenting images of differentbackground [8]. 


\section{EXPERIMENTAL RESULT AND DISCUSSION}

A variety of noise levels are used in the study of both medial and non-medical images, and an experiment is done on each set of images. The images from the following database are used to test the filtering each time. http://www.imageprocessingplace.com/DIP-3E/dip3e_book_images_downloads.htm five medical images, five non-medical images, and five color images are classified as three categories of images. Experiments are conducted using the MATLAB R2018b software, with the system configuration of $12 \mathrm{~Gb}$ ram and 64 bit windows operating syste
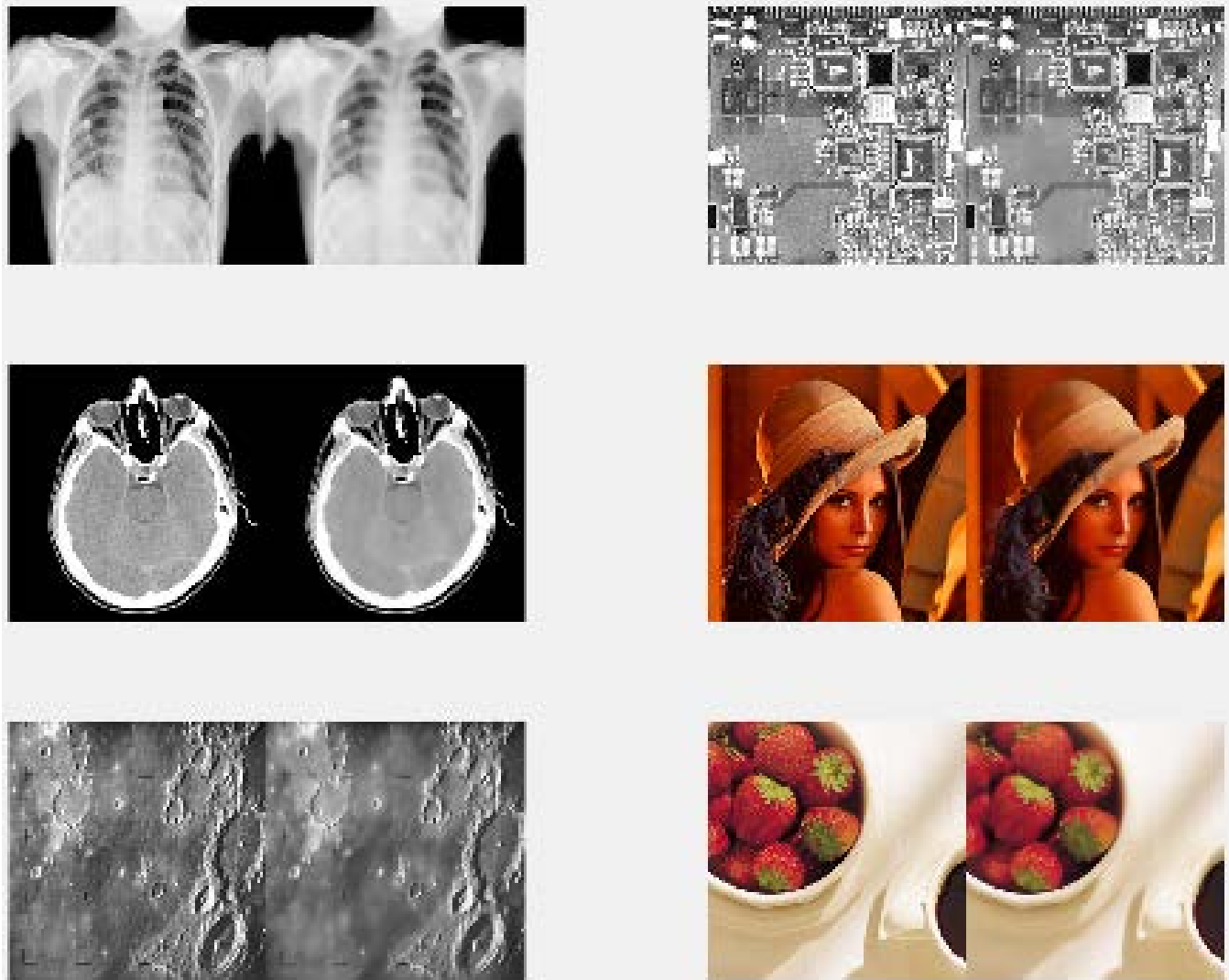

Figure1. Response of Guided filter 

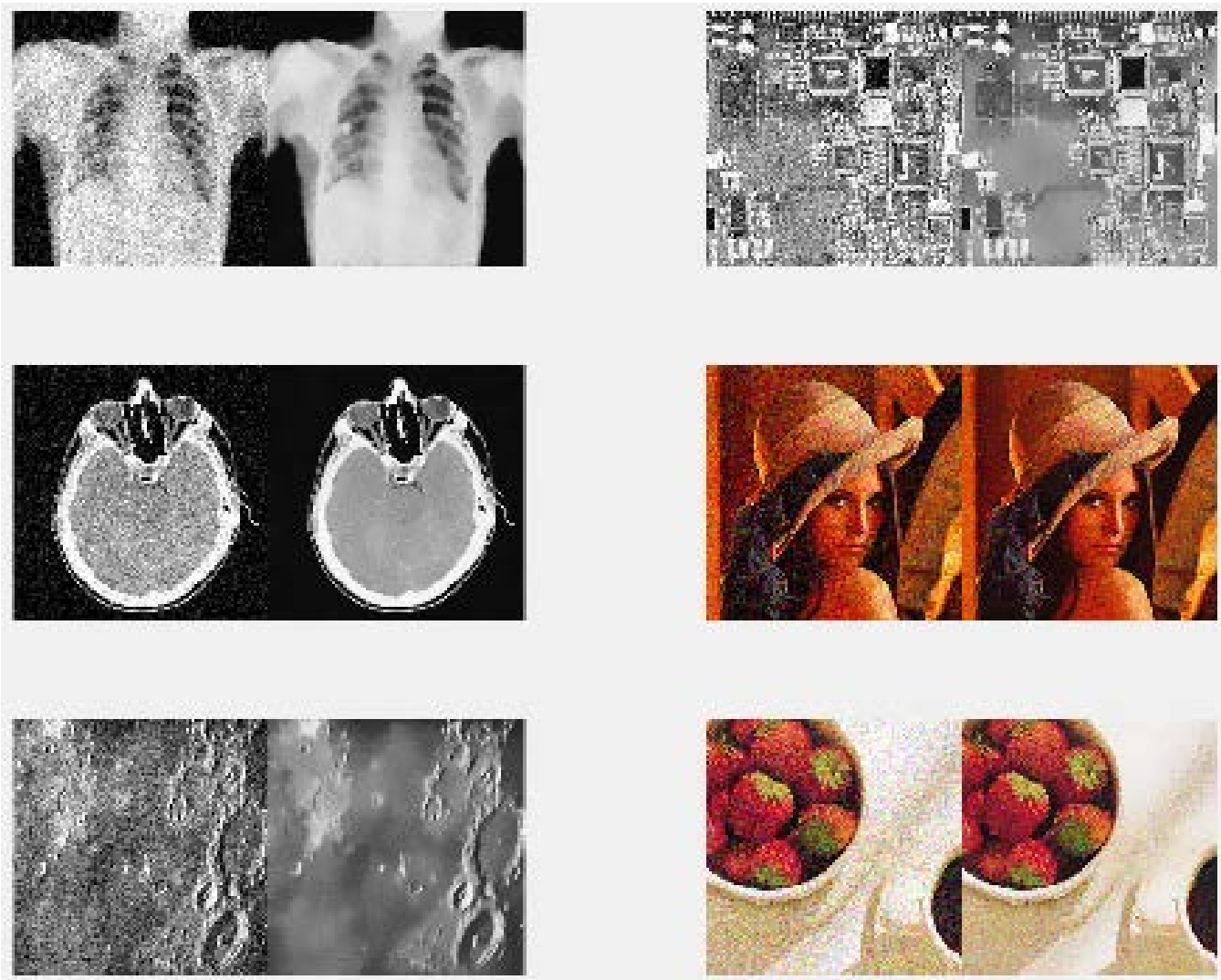

Figure 2 Response of Non-Local means filter
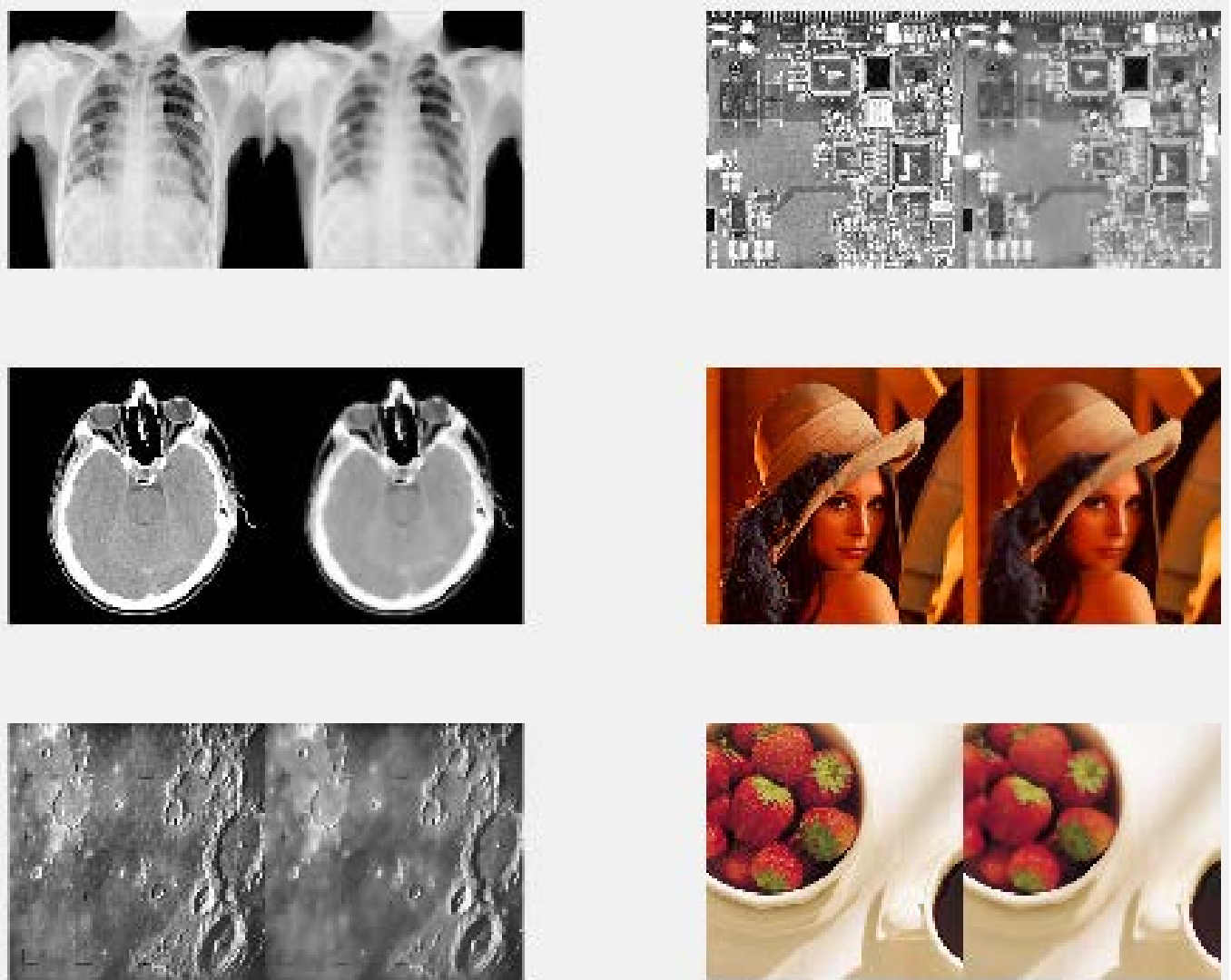

Figure 3 Response of Bilateral filter 

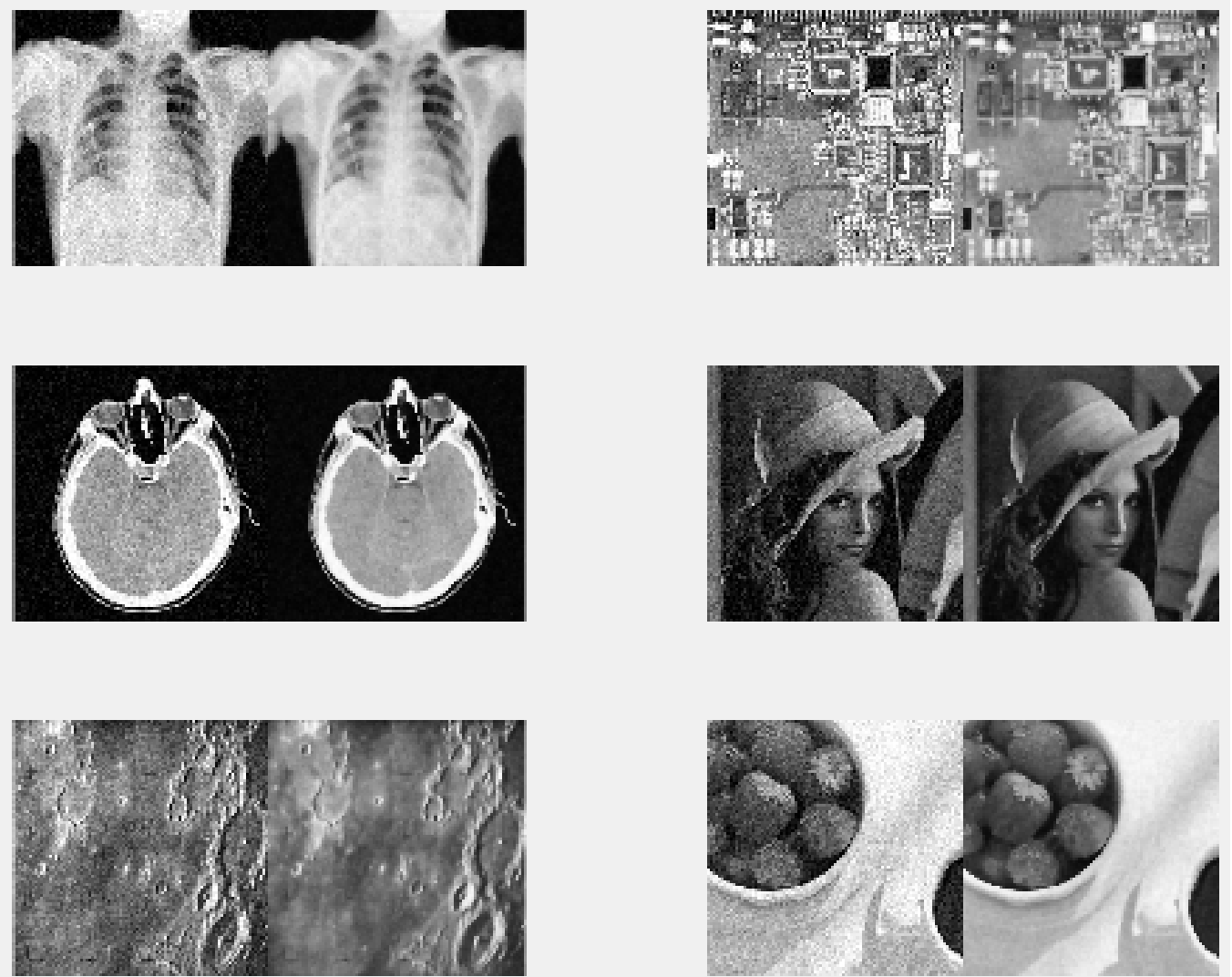

Figure 4 Response of Anisotropic diffusion filter
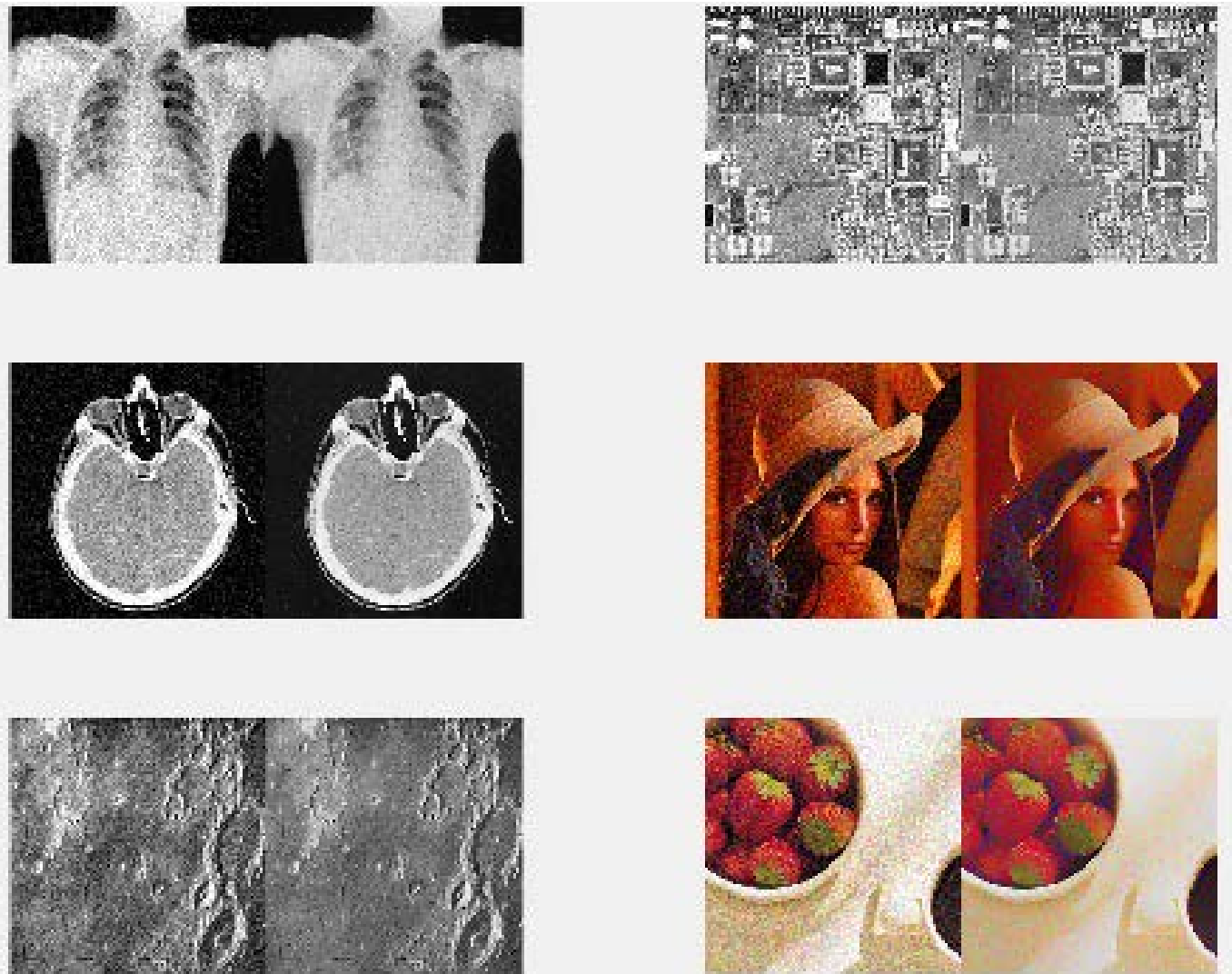

Figure 5 Response of Fast local Laplacian filter 

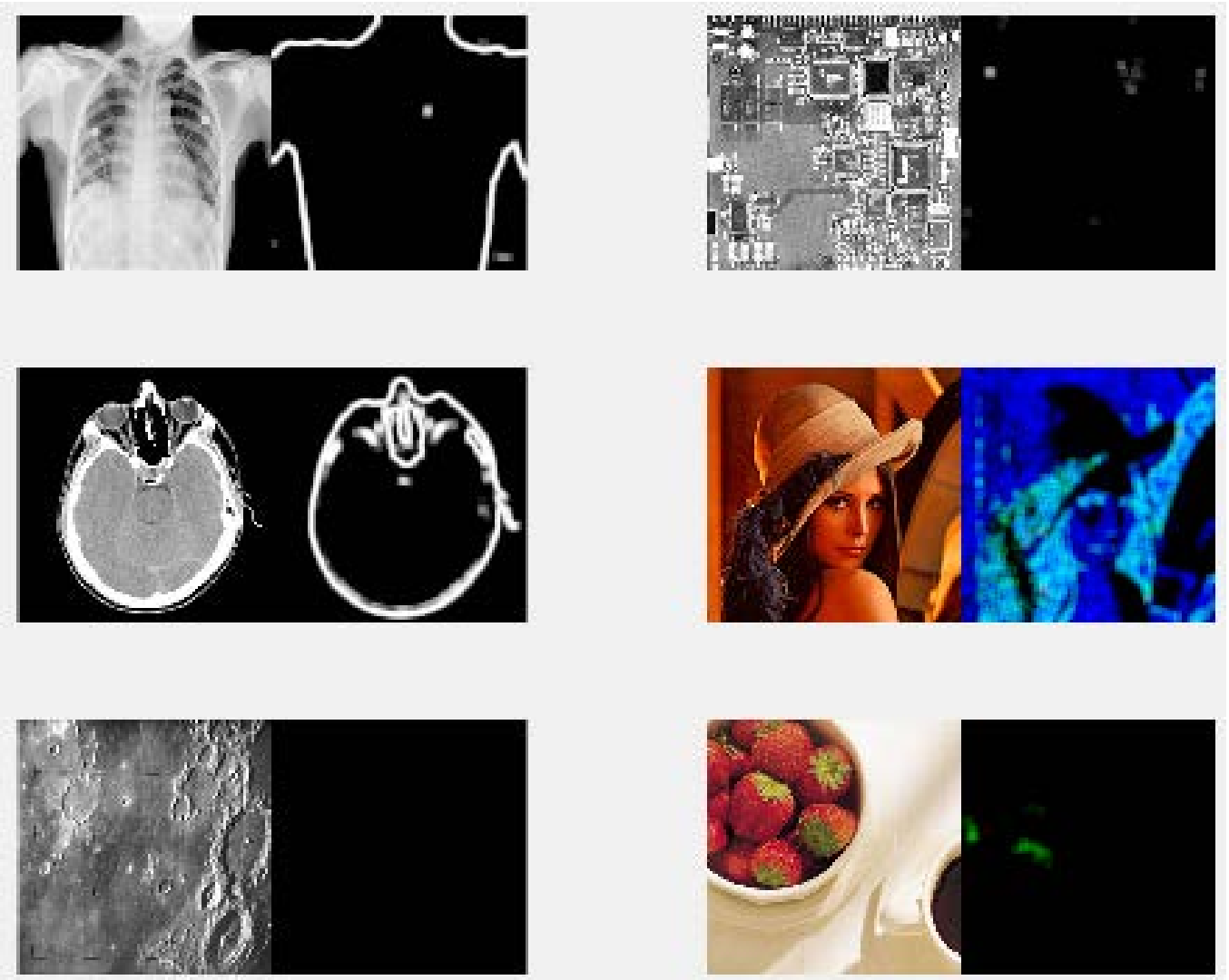

Figure 6 Response of Local entropy filter

Evaluation metrics used to compare the performance of each of the filter are, (i) Mean-squared error (MSE) - MSE measures the average squared difference between actual and ideal pixel values.(ii) Peak signal-to-noise ratio (PSNR)-PSNR is derived from the mean square error and indicates the ratio of the maximum pixel intensity to the power of the distortion, (iii) Structural similarity (SSIM) indexThe SSIM metric combines local image structure, luminance, and contrast into a single local quality score.

Table 1 Means squared error, Peak signal to noise ratio,Structural similarity index for each of filters.

\begin{tabular}{|c|c|c|c|c|c|c|}
\hline & \multicolumn{3}{|c|}{ Guided filter } & \multicolumn{3}{|c|}{ Non-Local means filter } \\
\hline Input Image & MSE & PSNR & SSIM & MSE & PSNR & SSIM \\
\hline chest-x ray & 20.86546 & 34.93652 & 0.9316 & 52.68915 & 30.91359 & 0.737607 \\
\hline HeadCT & 79.27853 & 29.13925 & 0.901431 & 61.4785 & 30.24357 & 0.85921 \\
\hline moon & 24.86079 & 34.17565 & 0.91439 & 34.76254 & 32.71969 & 0.768502 \\
\hline Circuitboard & 32.87178 & 32.96257 & 0.977847 & 8.202428 & 38.99138 & 0.988613 \\
\hline Lenna & 66.2605 & 29.91826 & 0.831451 & 73.73363 & 29.45415 & 0.754828 \\
\hline strawberries & 27.42361 & 33.74956 & 0.985488 & 18.26537 & 35.51452 & 0.949117 \\
\hline
\end{tabular}

\begin{tabular}{|c|c|c|c|c|c|c|}
\hline & \multicolumn{3}{|c|}{ Bilateral filter } & \multicolumn{3}{|c|}{ Anisotropic diffusion filter } \\
\hline Input Image & MSE & PSNR & SSIM & MSE & PSNR & SSIM \\
\hline chest-x ray & 26.70108 & 33.86552 & 0.921321 & 253.9648 & 24.08307 & 0.448877 \\
\hline Head CT & 544.2553 & 20.77278 & 0.699225 & 982.9769 & 18.20537 & 0.518537 \\
\hline moon & 186.2301 & 25.4303 & 0.838283 & 165.6869 & 25.93792 & 0.587094 \\
\hline Circuit board & 64.81202 & 30.01425 & 0.961182 & 278.3012 & 23.68565 & 0.4625 \\
\hline Lenna & 95.72693 & 28.32046 & 0.790268 & 457.248 & 21.52929 & 0.457015 \\
\hline strawberries & 52.50754 & 30.92859 & 0.979099 & 292.2807 & 23.4728 & 0.404075 \\
\hline
\end{tabular}




\begin{tabular}{|c|c|c|c|c|c|c|}
\hline & \multicolumn{3}{|c|}{ Fast local Laplacian filter } & \multicolumn{3}{|c|}{ Local entropy filter } \\
\hline Input Image & MSE & PSNR & SSIM & MSE & PSNR & SSIM \\
\hline chest-x ray & 233.8014 & 24.44233 & 0.782655 & 27243.76 & -44.3527 & 0.127328 \\
\hline Head CT & 169.1345 & 25.84848 & 0.868006 & 24867.08 & -43.9562 & $6.8 \mathrm{E}-09$ \\
\hline moon & 122.878 & 27.23606 & 0.558412 & 14971.33 & -41.7526 & 0.385736 \\
\hline Circuit board & 464.9957 & 21.45631 & 0.83086 & 9172.013 & -39.6246 & -0.00124 \\
\hline Lenna & 256.5822 & 24.03854 & 0.723388 & 14950.16 & -41.7465 & $1.7 \mathrm{E}-13$ \\
\hline strawberries & 241.4433 & 24.30265 & 0.950593 & 29252.4 & -44.6616 & $-4 \mathrm{E}-08$ \\
\hline
\end{tabular}

The performance of the Guided filter, non-Local means filter, Bilateral filter, Anisotropic diffusion filter, fast local Laplacian filter, and Local entropy filter is shown in Figures 1-6. Table 1 displays the MSE, PSNR, and SSIM of each filter on several images, including chest X-rays, head CTs, the moon, a circuit board, Lenna, and strawberries.

\section{CONCLUSION}

There are several spatial filters available for applications such as image smoothing, image sharpening, image enhancement, and noise filtering, among others. This article focuses on edge-preserving fillers, and the efficacy of each filter in eliminating noise was tested. Based on table 1, it is determined that the Guided filter, non-Local means filter, and Bilateral filter obtain PSNRs greater than 30 for nearly all input images. As a result of their noise reduction applications, these three filters can be utilized for further image analysis.

\section{REFERENCES}

[1] Kaiming He, Jian Sun, Xiaoou Tang. Guided Image Filtering. IEEE ${ }^{\circledR}$ Transactions on Pattern Analysis and Machine Intelligence, Volume 35, Issue 6, pp. 1397-1409, June 2013.

[2] Buades, A., B. Coll, and J.-M. Morel. "A Non-Local Algorithm for Image Denoising." 2005

IEEE ${ }^{\circledR}$ Computer Society Conference on Computer Vision and Pattern Recognition. Vol. 2, June 2005, pp. 60-65.

[3] Immerkær, J. "Fast Noise Variance Estimation." Computer Vision and Image Understanding. Vol. 64, Number 2, Sept. 1996, pp. 300-302.

[4] Tomasi, C., and R. Manduchi. "Bilateral Filtering for Gray and Color Images". Proceedings of the 1998 IEEE ${ }^{\circledR}$ International Conference on Computer Vision. Bombay, India. Jan 1998, pp. 836-846.

[5] Gerig, G., O. Kubler, R. Kikinis, and F. A. Jolesz. "Nonlinear anisotropic filtering of MRI data." IEEE Transactions on Medical Imaging. Vol. 11, No. 2, June 1992, pp. 221-232.

[6] Aubry, Mathieu, et al. Fast local Laplacian filters: Theory and applications. ACM Transactions on Graphics (TOG) 33.5 (2014): 167.

[7] Gonzalez, R. C., R. E. Woods, and S. L. Eddins. Digital Image Processing Using MATLAB. New Jersey, Prentice Hall, 2003, Chapter 11.

[8] Yang W, Cai L, Wu F, Image segmentation based on gray level and local relative entropy two dimensional histogram. PLoS ONE 15(3): e0229651,2020.

[9] Burger W., Burge M.J. Edge-Preserving Smoothing Filters. In: Digital Image Processing. Texts in Computer Science. Springer, London. 2016. https://doi.org/10.1007/978-1-4471-6684-9_17

[10] https://in.mathworks.com/help/images/linear-filtering.html?s tid=CRUX topnav

[11] http://www.imageprocessingplace.com/DIP-3E/dip3e_book_images_downloads.htm non-medical database for performance evaluation 